\title{
Thrombotic events in SARS-CoV-2 patients: an urgent call for ultrasound screening
}

\author{
Guido Tavazzi ${ }^{1,2^{*}}$, Luca Civardi², Luca Caneva² ${ }^{2}$ Silvia Mongodi and Francesco Mojoli
}

๑ 2020 Springer-Verlag GmbH Germany, part of Springer Nature

\section{Dear Editor,}

The COVID-19 outbreak in Wuhan city, China, in December 2019 has rapidly spread up to the level of Pandemia at the beginning of March 2020. A recent report from China has identified high inflammatory status as a predictor of adverse outcome, suggesting that mortality might be due to virally driven hyper-inflammation status [1].

There is a well-established link between inflammation and increased risk of deep vein thrombosis (DVT). A potential explanation is that vessel wall inflammation initiates thrombus formation, through the activation of endothelial cells, platelets, and leukocytes that trigger the coagulation pathway $[2,3]$.

Additionally, procoagulant state has long been recognized also as part of ARDS pathophysiology, demonstrated by the identification of diffuse pulmonary endothelial injury associated with platelets' activation, macro- and micro-thrombi thought to be either embolic, formed in situ or both [4]. Moreover, the interaction pathway among platelets, neutrophils, and endothelial cells' dysfunction in ARDS has been associated with deep vein thrombosis development [2]. High prevalence of acute pulmonary embolism (APE) has been recently reported in patients admitted with COVID-19-related pneumonia [5].

We report the prevalence of venous thrombotic events in patients consecutively admitted to the ICU of a Hub Hospital for SARS-CoV-2 since the beginning of the Italian outbreak infection on February 21, 2020. Informed consent was collected following the ad hoc procedures

\footnotetext{
*Correspondence: guido.tavazzi@unipv.it

${ }^{1}$ Department of Clinical-Surgical, Diagnostic and Pediatric Sciences, Unit of Anaesthesia and Intensive Care, University of Pavia, Pavia, Italy

Full author information is available at the end of the article
}

defined by the Ethics Committee for the COVID-19 pandemic.

All patients were sedated, mechanically ventilated and treated with prophylactic low-molecular-weight heparin (LMWH) adjusted on body weight since the admission.

Eight out of 54 patients (14.8\%) were diagnosed with deep vein thrombosis, 6 of which were central catheterrelated. Additionally, one patient had a thrombotic formation attached to the tricuspid valve in the absence of predisposing factors. Sub-segmental pulmonary embolism was found in two patients examined with computed tomography pulmonary angiography (CTPA) and one patient died of cardiac arrest with pulseless electrical activity (PEA) as presentation rhythm and sudden right ventricular dilatation.

In our experience $22.2 \%$ of patients $(83 \%$ male, $68 \pm 7$ years old; BMI $29.3 \pm 4.4 \mathrm{~kg} / \mathrm{m}^{2}$; C-reactive protein $25.7 \pm 9.2 \mathrm{mg} / \mathrm{dl}$, fibrinogen $657.1 \pm 200.6 \mathrm{mg} / \mathrm{dl}$ ) admitted to ICU due to SARS-CoV-2 interstitial pneumonia had venous thrombotic events (Fig. 1).

ICU admission and ARDS are considered both predisposing factors for a number of reasons, including the need for prolonged immobilization and hyper-inflammatory state. The prevalence of vein thrombosis in patients admitted with ARDS is currently unknown.

A recent report showed that 10 out of 25 patients admitted with pneumonia due to COVID-19 presented sub-segmental APE, assessed with CPTA. No associated deep vein thrombosis was reported as the authors did not perform ultrasound screening.

Because of the pathophysiological link between inflammation and thrombosis development [3], especially in critically ill patients, the hyper-inflammatory status of patients with COVID-19 [1] and the high prevalence of APE [5] and of vein thrombotic events found in our population, we strongly suggest that a close vein ultrasound screening and monitoring should be performed in all

\section{6 Springer}




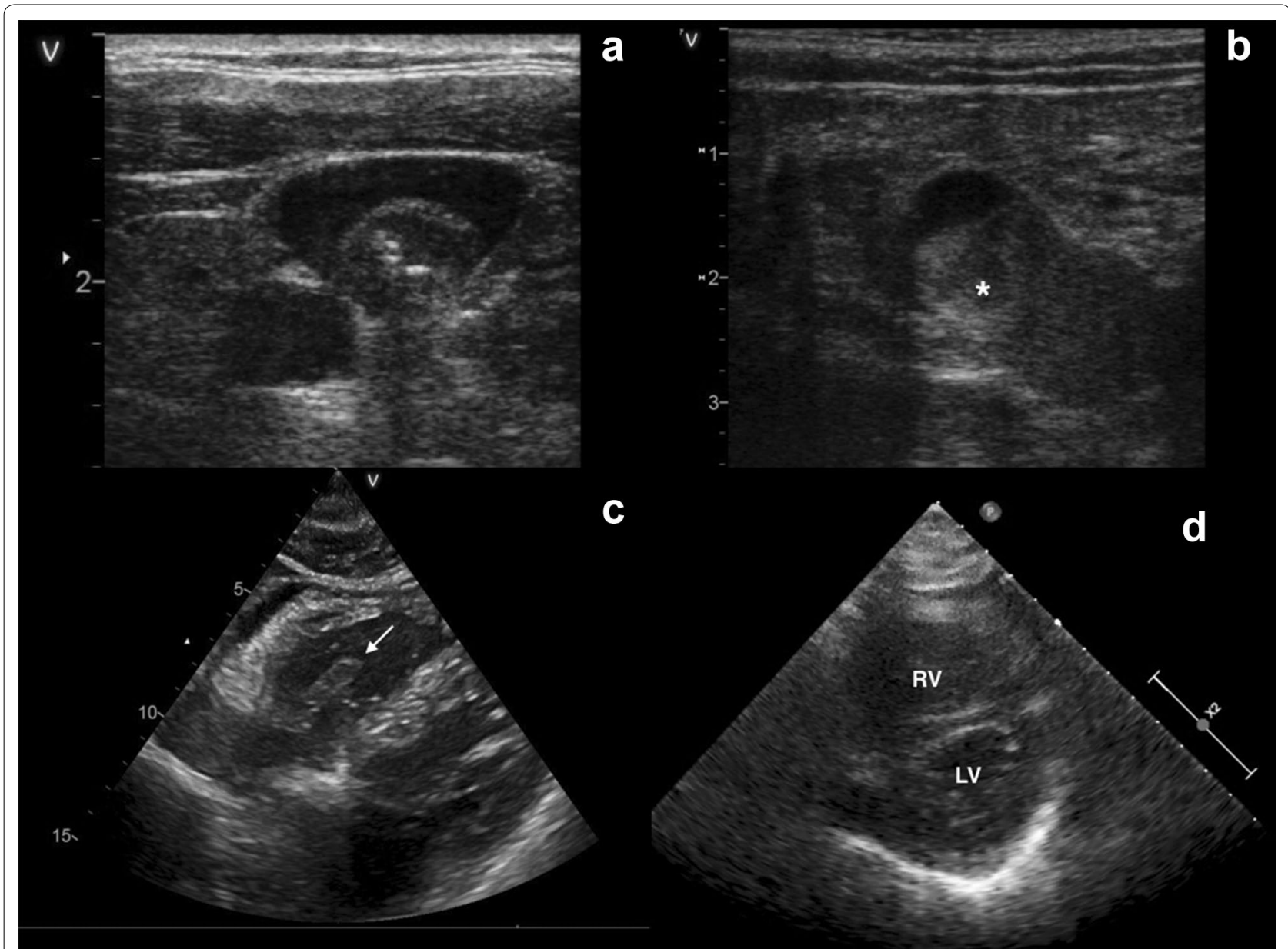

Fig. 1 Ultrasound demonstration of thrombotic events in patients admitted with SARS-Cov2. Deep vein ultrasound showing (a-ESM1) the internal jugular vein with a thrombus surrounding a central catheter (central white point) and (b-ESM2) a thrombus of superficial and common femoral vein (white asterisk). Transthoracic echocardiography subcostal view demonstrating (c-ESM3) a thrombus in the right ventricular cavity, attached to the tricuspid valve (white arrow) and (d-ESM4) parasternal short axis view in patient with right ventricular dilatation (right ventricular/ left ventricular area 0.7) inducing interventricular paradoxical septal motion

patients hospitalized due to SARS-CoV-2-related infection. Additionally, right ventricular dilatation/dysfunction should trigger the suspicion of APE.

\section{Electronic supplementary material}

The online version of this article (https://doi.org/10.1007/s00134-020-06040-3) contains supplementary material, which is available to authorized users.

\section{Author details}

1 Department of Clinical-Surgical, Diagnostic and Pediatric Sciences, Unit of Anaesthesia and Intensive Care, University of Pavia, Pavia, Italy. ${ }^{2}$ Anesthesia and Intensive Care, Fondazione Policlinico San Matteo Hospital IRCCS, Pavia, Italy.

\section{Acknowledgements}

We thank all the nurses and physicians involved in the management of such epidemics and the Pavia COVID-19 Task Force (Dr. Marena Carlo; Dr. Calvi Monica; Dr Grugnetti Giuseppina; Dr Maurelli Marco; Dr Muzzi Alba; Prof. Raffaele Bruno; Dr Lago Paolo; Prof. Marseglia Gianluigi; Prof. Perlini Stefano; Dr Palo Alessandra; Prof Baldanti Fausto; Prof. Corsico Angelo Guido; Prof. Di
Sabatino Antonio, Prof. lotti Giorgio; Prof. Benazzo Marco, Prof.Carlo Nicora, Prof.Antonio Triarico and Dr. Vincenzo Petronella).

\section{Authors' contributions}

All authors contributed to data collection and redaction, writing and final revision before submission of the paper.

\section{Funding}

No external funding was used, authors used institutional fundings. The corresponding author had full access to the data and had final responsibility for the decision of submitting for publication.

\section{Compliance with ethical standards}

\section{Conflicts of interest}

FM received fees for lectures from GE Healthcare, Hamilton Medical, SEDA SpA outside the present work. SM received fees for lectures from GE Healthcare, outside the present work. GT received fees for lectures by GE Healthcare, outside the present work. 


\section{Publisher's Note}

Springer Nature remains neutral with regard to jurisdictional claims in published maps and institutional affiliations.

\section{Accepted: 4 April 2020}

Published online: 22 April 2020

\section{References}

1. Ruan Q, Yang K, Wang W, Jiang L, Song J (2020) Clinical predictors of mortality due to COVID-19 based on an analysis of data of 150 patients from Wuhan, China. Intensive Care Med. https://doi.org/10.1007/s0013 4-020-05991-x

2. Frantzeskaki F, Armaganidis A, Orfanos SE (2017) Immunothrombosis in acute respiratory distress syndrome: cross talks between inflammation and coagulation. Respiration 93(3):212-225
3. Saghazadeh A, Hafizi S, Rezaei N (2015) Inflammation in venous thromboembolism: cause or consequence? Int Immunopharmacol 28(1):655-665

4. Tomashefski JF Jr, Davies P, Boggis C, Greene R, Zapol WM, Reid LM (1983) The pulmonary vascular lesions of the adult respiratory distress syndrome. Am J Pathol 112(1):112-126

5. Helms J, Tacquard C, Severac F, Leonard-Lorant I, Ohana M, Delabranche X, Merdji H, Clere-Jehl R, Schenck M, Fagot Gandet F, Fafi-Kremer S,

Castelain V, Schneider F, Grunebaum L, Anglés-Cano E, Sattler L, Mertes P-M, Meziani F, and for the CRICS TRIGGERSEP Group (Clinical Research in Intensive Care and Sepsis Trial Group for Global Evaluation and Research in Sepsis) (2020) High risk of thrombosis in patients in severe SARS-CoV-2 infection: a multicenter prospective cohort study. Intensive Care Med. https://doi.org/10.1007/s00134-020-06062-x 